\title{
Lessons Learned from Evaluating the Usability of Mobile Spreadsheet Applications
}

\author{
Derek Flood, Rachel Harrison, and Claudia Iacob \\ Oxford Brookes University \\ Gipsy Lane, Oxford, United Kingdom \\ \{derek.flood, rachel.harrison, iacob\} abrookes.ac.uk
}

\begin{abstract}
It is estimated that $90 \%$ of all the analysts in business perform calculations on spreadsheets. Due to advances in technology, spreadsheet applications can now be used on mobile devices and several such applications are available for platforms such as Android and iOS. Research on spreadsheets revolves around several themes, but little work has been done in evaluating the usability of such applications (desktop or mobile). This paper presents lessons learned and usability guidelines derived from laboratory usability testing of mobile spreadsheet applications. Twelve participants were given a task to be solved using a mobile spreadsheet application and based on the video recordings of their interactions with the application patterns of recurring actions and sequences of actions were derived. Navigation, selection, feedback, and transparency of features were some of the main themes in the results of the testing, pointing to a set of guidelines which are also generalizable across other types of mobile applications.
\end{abstract}

Keywords: Mobile applications, usability guidelines.

\section{Introduction}

Spreadsheets are widely used for tasks such as inventory administration, educational applications, scientific modeling, financial systems, etc. [1]. It is estimated that $90 \%$ of all analysts in business perform calculations on spreadsheets [2], the financial business being the domain where spreadsheets are most used. Due to advances in technology spreadsheet applications may be used on mobile devices, several such applications being available for platforms such as Androids or iOS. A recent study has shown that $79 \%$ of the participants (all recruited through the European Spreadsheet Risk Interest Group) required access to a spreadsheet while away from a desktop computer, mostly in the following contexts: a) daily commute, b) demonstrating data to clients, c) inbound email onto a mobile device, d) discussing urgent changes with coworkers [3]. However, the majority of the participants used mobile spreadsheet applications less than once a month, mostly because of the usability challenges such applications bring.

Research on spreadsheets has focused on various aspects, including expanding spreadsheet language through direct manipulation and gestures [4], communicating unit error messages in spreadsheets [5], type inferences for spreadsheets [6], 
graph-based visualizations [7], testing [8], and web based spreadsheet-mediated collaboration [9]. However, little work has been done in identifying the usability issues revolving around spreadsheet applications (mobile or desktop-based) and ways to go about resolving them. To address this gap, this paper describes the results of a series of human-centered laboratory usability tests performed on mobile spreadsheet applications, pointing to some of the lessons learned and guidelines applicable to the mobile context design.

\section{Related Work}

Zhang and Adipat highlighted a number of issues that affect the usability of mobile applications, such as the mobile context, small screen size and different display resolutions, limited processing capability and power and tedious data entry methods [10]. Efforts have been made to look into some of these issues, focusing mostly on mobile web browsing [11, 12, 13] and mobile guides [14]. Schmield et al. describe the results of a multidimensional study which investigates usage scenarios as well as the usability of mobile tailored websites, answering questions such as: what kinds of websites are most often accessed using a mobile phone and who are the users etc. [12]. The findings show (for example) that the typical mobile phone user is male, between 20 and 29, and of the mobile tailored sites investigated, 55\% fell in the category 'Information services', while $20 \%$ were social networking sites.

A discussion on the optimal information hierarchy for mobile use and the usability effects for reduced screen size is presented in [11]. Slow reading speed, poor comprehension and poor information retrieval performance are just a few of the usability effects reduced screen size brings [15]. Ways of dealing with such issues include adapting the way information is structured in hierarchies [11]. A comparative study of four hierarchies on three different devices found that users preferred narrower hierarchies on all the devices. In [16], a comparative study of zoomable UIs (with and without overviews) used in the context of mobile maps, diagrams, and web pages shows that for zoomable UI overviews the "effectiveness in search tasks is highly dependent on the type of information overviews can provide and on the structure of the considered space". For example, applications involving maps with dynamic content could benefit from zoomable UIs with overviews. The results of a usability study in which users' mobile web browsing experience was evaluated in comparison to desktop web browsing have also been described [13]. All the participants managed to complete all the tasks given to them on the desktop, but only one participant achieved this on the mobile device. Moreover, $80 \%$ of the time was dedicated to completing the tasks on the mobile device. Also subjects found it more difficult to correct their mistakes when using the mobile device. Overall, even if the subjects found the changes of the usual page structure on the mobile device easy to understand and learn, they experienced difficulties while interacting with it and this affected their satisfaction. 


\section{Evaluating the Usability of Mobile Spreadsheets}

For evaluating the usability of mobile spreadsheet applications, laboratory usability tests were run with 12 participants, whose actions were video recorded, coded, and further analyzed. The participants were given the task of editing nine cells and locating seven pieces of information located on a given spreadsheet using an iPhone device (which had the Spreadsheet app installed, the most currently used and representative spreadsheet mobile app). Out of the 12 participants, $75 \%$ of the participants rated their level of experience with spreadsheets as being intermediate. Only 1 participant $(8.33 \%)$ rated their level of experience as being novice. As far as experience with the iPhone was concerned, $60 \%$ of participants rated themselves as either intermediate or expert. With the exception of one participant, the remaining participants all had experience on other smart phone devices. In terms of age, the majority of participants (42\%) were between the age of 21 and 30. During the laboratory tests, the actions of the participants were video recorded while using the mobile spreadsheet application. These recordings were coded for analysis by associating each action with one of the codes depicted in Figure 1. The most often performed action while using the mobile spreadsheet application was scrolling from left to right. This may be explained by two points: 1) Zooming out was performed infrequently (less than $2 \%$ of the video fragments being assigned this code) which led to the need to navigate within a spreadsheet using scrolling and 2) There was very little feedback from the application on the participants' actions, so the participants were at times uncertain as to which part of the spreadsheet they were viewing and how this part was related to the rest of the spreadsheet. Navigation was common also between screens, the participants switching from one spreadsheet to another quite frequently.

Figure 1 highlights the high percentage of actions coded with "sameCellSelect" (i.e. the user clicks on the same cell they clicked on during the previous action) and "wrongCellSelect" (i.e. the user clicks on a different cell than the one they intended to click on). The discussion would also include "multipleCellSelect" (i.e. the user clicks on multiple cells at once by mistake) even if the percentage for this is much lower. What often happened was that the participants would aim to select one cell, but they were either selecting another cell close to the one desired or they were not getting any feedback on the action so they tried to select the same cell again to ensure the cell was selected. The percentage of actions coded with "newCellSelect" (i.e. the user clicks on the right cell at a first attempt) was just a little bit higher than "sameCellSelect". Particularly important, "actionFeedback" would mark the times when the participants perceived and were supported by feedback from the application on their interactions with it (e.g. trying to close one spreadsheet, they would be asked for confirmation). However, such occurrences were rare, the distribution of such actions being the lowest. Even so, the participants (with few exceptions) did not give up the task or parts of it. 


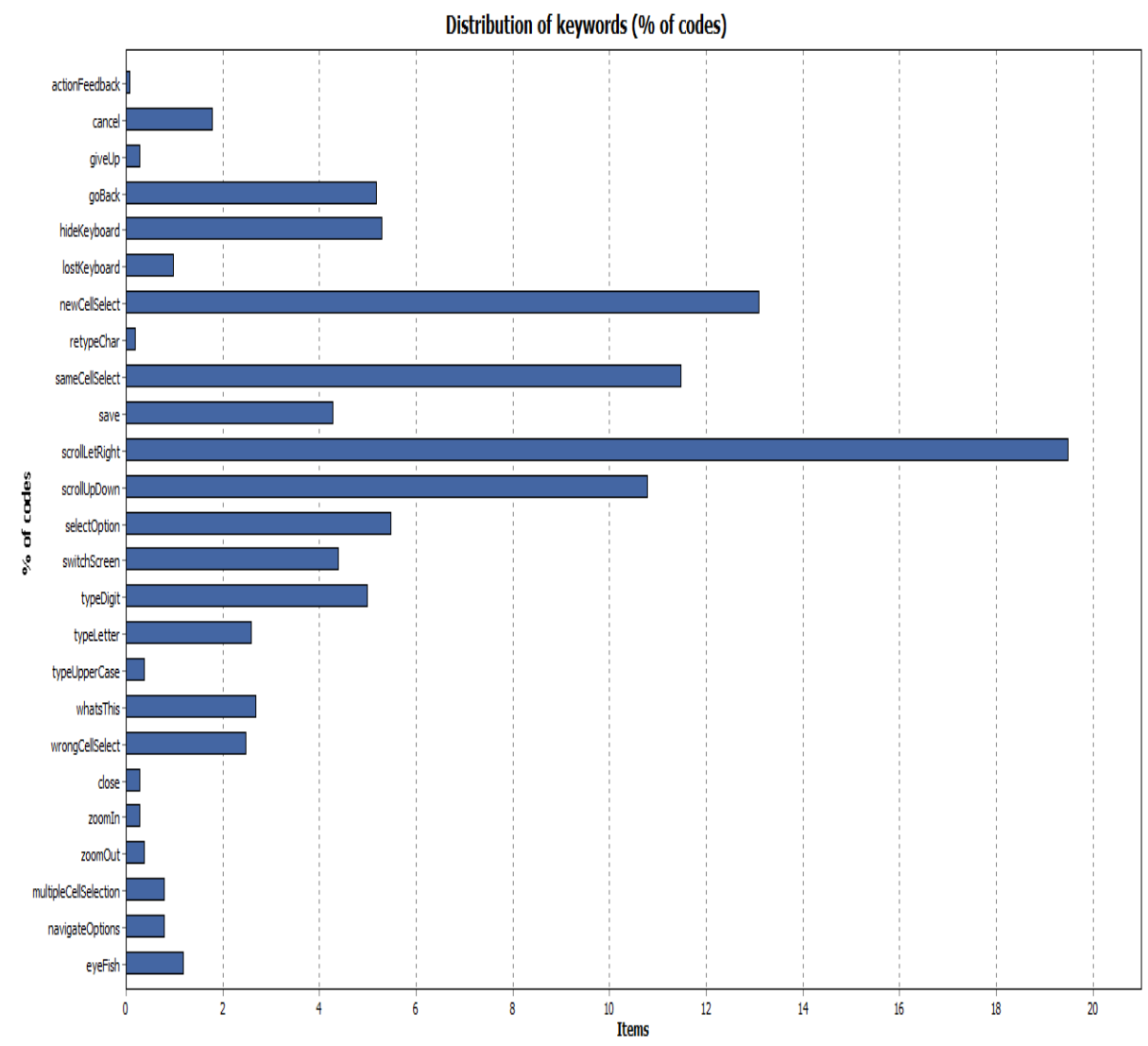

Fig. 1. Action codes distribution for the lab tests

"Whatsthis" was used to code actions during which the participants were puzzled by the answer of the application to their actions. Apart for this, the system provided very little feedback, and lacked support for issues such as: a) was the action successful (e.g. Is the background color set?), b) what are the consequences of an action, especially if it was made by mistake (e.g. Selecting the wrong cell), c) where is the cursor located, etc. There was very little use of features such as fisheye, zoom in and zoom out.

In addition to analyzing the frequency of each individual action, we also analyzed the common sequences of actions (Figure 2). Some recurring sequences of actions participants performed bring to light potential issues with the application itself. The act of Saving changes indicates that the participant successfully made the desired modifications. In more than a half of the cases, this led to navigating the spreadsheet further on. Navigation was mostly performed through scrolling, zooming actions being performed rarely. In $37.9 \%$ of the cases, scrolling from left to right led to more scrolling. This is partially explained by the fact that most of the time a scroll to the left would immediately be followed by a scroll to the right and vice versa. Similarly, scrolling up and down led to more scrolling. Also, not surprisingly, switching to another screen mostly led to navigating within the screen through scrolling actions. 


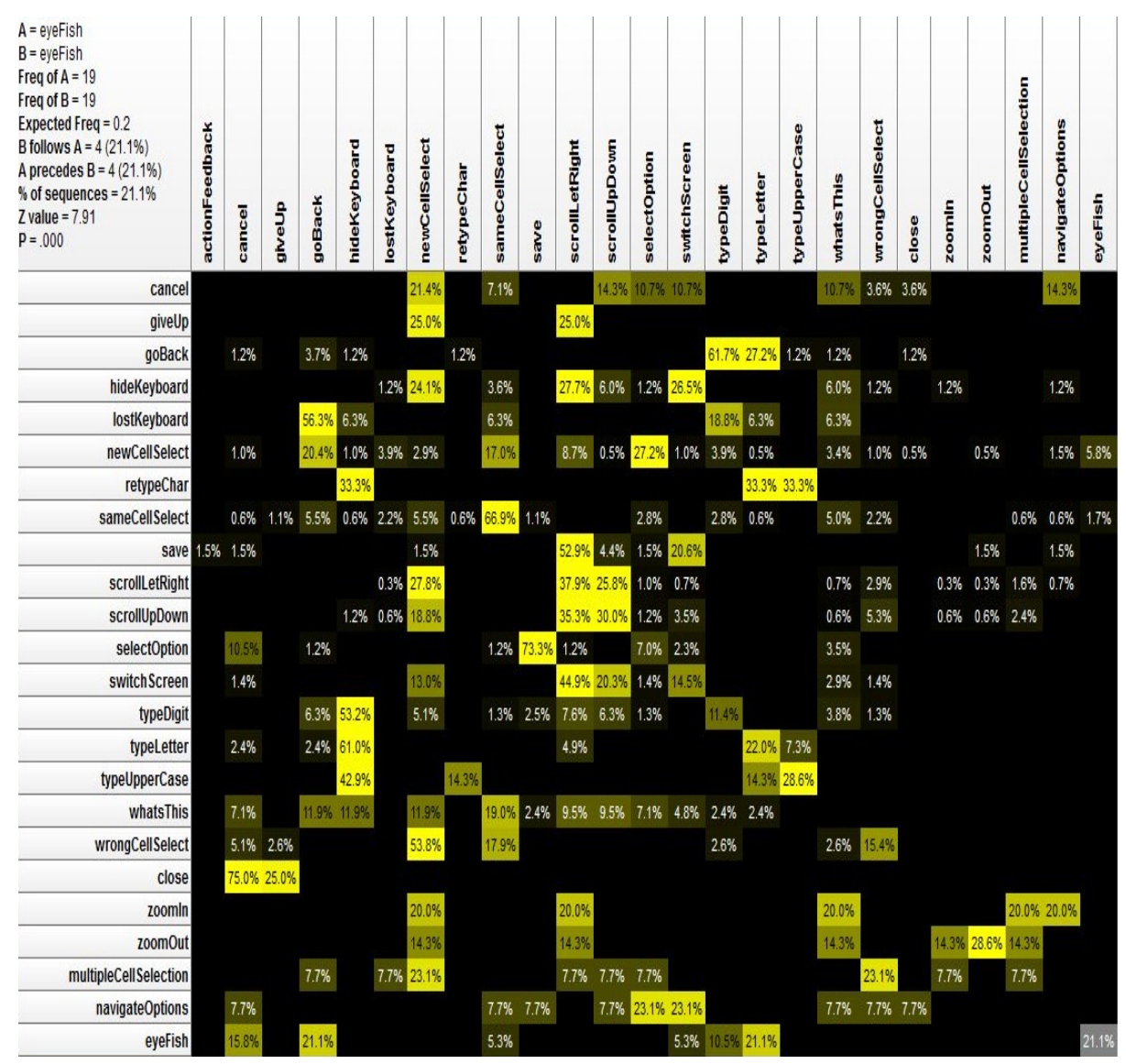

Fig. 2. Sequence code distribution for the lab tests

"SameCellSelect" led to the same action in $66.9 \%$ of the cases, showing that selecting the same cell over and over again was common. Due to lack of feedback from the application, the participants would select the same cell several times in a row. The maximum repetition of this action was 9 times. Right after selecting a wrong cell (different from the one desired), the participants would select the one desired in more than half of the cases. However, $17.9 \%$ percent of the cases point to the situation where the same cell (the wrong one) was selected. Selecting an option (e.g. selecting the background color for a cell) led to saving the modifications in $73.3 \%$ of the cases. However, at times, selecting an option would lead to reselecting that particular option. This was partially because the application would provide no feedback leading to confusion on the completion of the action. As an answer to that, the participants would at times try to repeat the action in order to check or validate that the action had been performed. $75 \%$ of the cases when the participants intended to close the application were followed by a cancelation of the action. This was particularly interesting since in most cases it was not clear to the participants that the 
close button would close the whole application and not just the open spreadsheet. Only when asked whether they want to save the work (as result of closing), did it become clear that the close button closes the application entirely. In the majority of cases, the participants would hide the keyboard right after typing some characters because otherwise the keyboard would impede the navigation within the spreadsheet. Preceding "whatsthis" coded actions were: a) zooming actions (the participants were uncertain of what caused the zooming and how to go back to the previous state) and b) canceling an action (without any feedback the participants sometimes became confused).

To determine the level of satisfaction participants had, they were asked to complete a SUS questionnaire, the average overall SUS score for the spreadsheet app being $50.42 \%$. In addition to the low score, when asked if they would use the mobile spreadsheet application again, only half of the participants said that they would.

\section{Lessons Learned, Guidelines, and Discussion}

Based on direct observation and on the results drawn from the study, this section describes a set of 4 guidelines and lessons learned.

1. Make navigation features obvious. The laboratory tests showed that features such as fisheye, or zooming in and/or out were rarely used, leading to poor navigation and overall awareness. Most of the participants were not even aware of the existence of these features. Such features should be clearly signaled such that the user is aware of the full potential of the application.

2. Provide feedback. Feedback supports users in understanding their actions and the impact of their actions in relation to the application. During the study, five types of needed feedback became evident.

a) Validation feedback. Once the user performs an action on the application, it is helpful for them to understand whether that was a correct action with respect to the interaction context they are in. For example, selecting a spreadsheet cell should be accompanied by feedback on whether the cell was the right cell or not.

b) Location feedback. Due to the small size of mobile devices, it is easy for users to get disoriented with respect to the cursor's location on the screen or to the position of the part they are visualizing in the overall document For such disorientation to be avoided, it is important for the user to be provided with feedback on the location of what they are currently viewing in the overall picture.

c) Neighborhood feedback. Participants needed to navigate through the document for extensive periods of time also to become aware of the data contained by the cells surrounding those visible on the screen. Feedback on such information would reduce the users' navigation overload.

d) Selection feedback. One of the main issues observed during the study was that the participants were not always sure that the cell they were trying to select was indeed selected. In answer to that, they kept selecting the same cell repeatedly. In part, this problem could be solved by providing feedback once a cell is selected by 
highlighting its margins or bringing the cell to the front. Similar techniques could be used for applications which use other types of selections.

e) Keyboard feedback. The location of the keyboard was not always clear to the participants. At times, they struggled to understand how to hide the keyboard or, on the contrary, how to make it accessible. The application should support the user by providing feedback on the current state of the keyboard at all times.

3. Hints as to the consequences of each action. Users might be unaware of the consequences of some actions and realize only after the action has been performed what it led to. Even worse, in some cases users might not perceive the answer of the application to their action or fail to understand it. Hints on what each action led to would help users. As a concrete example, the color changing of a cell in the spreadsheet application could be modeled as a wizard where all the steps are specified a priori and the user is aware of what the next step is.

4. Provide evident milestones. At times during the study, participants faced difficulties in trying to cancel an action by clicking mistakenly on the button closing the application. It was not always clear how to save, cancel or quit the application and this affected their interaction with the application. Such features should be made evident and distinct, so that at any time during his/her interaction with the application the user is aware of how such features work and how they are different.

\section{Conclusions}

In this paper, we looked at the results obtained through a pilot study addressing the usability evaluation of mobile spreadsheet applications. We learned that several types of feedback are particularly important when interacting with a mobile application, some of them being feedback on the state and location of the keyboard, feedback on the location of the cursor and on the currently viewed part of the overall document. Also, navigation features should be made evident since only experienced users made any use of zooming or fisheye features. Functions such as saving the work done, closing the application, or canceling an action should be clear and the difference should be made clear to the users. Our results will indicate to mobile spreadsheet application designers some of the difficulties and challenges users face while using such applications.

\section{References}

1. Hermans, F., Pinzger, M., van Deursen, A.: Supporting professional spreadsheet users by generating leveled dataflow diagrams. In: Proceedings of ICSE 2011, pp. 451-460 (2011)

2. Winston, W.L.: Executive education opportunities. OR/MS Today 28(4) (2001)

3. Flood, D., Harrison, R., McDaid, K.: Spreadsheets on the move: An evaluation of mobile spreadsheets. In: The European Spreadsheet Risk Interest Group Annual Conference (2011) 
4. Burnett, M.M., Gottfried, H.: Graphical definitions: expanding spreadsheet languages through direct manipulation and gestures. ACM Trans. Com-Hum. Interact. 5(1), 1-33 (1998)

5. Abraham, R., Erwig, M.: How to communicate unit error messages in spreadsheets. In: Proceedings of the First Workshop on End-user Software Engineering, pp. 1-5 (2005)

6. Abraham, R., Erwig, M.: Inferring templates from spreadsheets. In: Proceedings of ICSE 2006, pp. 182-191 (2006)

7. Kankuzi, B., Ayalew, Y.: An end-user oriented graph-based visualization for spreadsheets. In: Proceedings of the 4th International Workshop on End-user Software Engineering, pp. 86-90 (2008)

8. Rothermel, G., Burnett, M., Li, L., Dupuis, C., Sheretov, A.: A methodology for testing spreadsheets. ACM Trans. Softw. Eng. Methodol. 10(1), 110-147 (2001)

9. Ginige, A., Paolino, L., Sebillo, M., Shrodkar, R., Vitiello, G.: User requirements for a web based spreadsheet-mediated collaboration. In: Proceedings of AVI 2010, pp. 133-136 (2010)

10. Zhang, D., Adipat, B.: Challenges, Methodologies, and Issues in the Usability Testing of Mobile Applications. International Journal of Hum-Comp. Interaction 18(3), 293-308 (2005)

11. Geven, A., Sefelin, R., Tscheligi, M.: Depth and breadth away from the desktop: the optimal information hierarchy for mobile use. In: Proceedings of MobileHCI 2006, pp. 157-164 (2006)

12. Schmiedl, G., Seidl, M., Temper, K.: Mobile phone web browsing: a study on usage and usability of the mobile web. In: Proceedings of MobileHCI 2009 (2009)

13. Shrestha, S.: Mobile web browsing: usability study. In: Proceedings of Mobility 2007, pp. 187-194 (2007)

14. Kjeldskov, J., Graham, C., Pedell, S., Vetere, F., Howard, S., Balbo, S., Davies, J.: Evaluating the usability of a mobile guide: the influence of location, participants and resources. Behaviour and Information Technology 24(1), 51-65 (2005)

15. Jones, M., Marsden, G., Mohd-Nasir, N., Boone, K., Buchanan, G.: Improving Web Interaction on Small Displays. In: Proc. WWW-8, pp. 1129-1137 (1999)

16. Burigat, S., Chittaro, L., Parlato, E.: Map, diagram, and web page navigation on mobile devices: the effectiveness of zoomable user interfaces with overviews. In: Proceedings of MobileHCI 2008, pp. 147-156 (2008) 